\title{
Increased isobutanol production in Saccharomyces cerevisiae by eliminating competing pathways and resolving cofactor imbalance
}

\author{
Fumio Matsuda ${ }^{1,2,3}$, Jun Ishii ${ }^{2}$, Takashi Kondo ${ }^{2,4}$, Kengo Ida ${ }^{5}$, Hironori Tezuka ${ }^{5}$ and Akihiko Kondo ${ }^{3,5^{*}}$
}

\begin{abstract}
Background: Isobutanol is an important target for biorefinery research as a next-generation biofuel and a building block for commodity chemical production. Metabolically engineered microbial strains to produce isobutanol have been successfully developed by introducing the Ehrlich pathway into bacterial hosts. Isobutanol-producing baker's yeast (Saccharomyces cerevisiae) strains have been developed following the strategy with respect to its advantageous characteristics for cost-effective isobutanol production. However, the isobutanol yields and titers attained by the developed strains need to be further improved through engineering of S. cerevisiae metabolism.
\end{abstract}

Results: Two strategies including eliminating competing pathways and resolving the cofactor imbalance were applied to improve isobutanol production in S. cerevisiae. Isobutanol production levels were increased in strains lacking genes encoding members of the pyruvate dehydrogenase complex such as LPD1, indicating that the pyruvate supply for isobutanol biosynthesis is competing with acetyl-CoA biosynthesis in mitochondria. Isobutanol production was increased by overexpression of enzymes responsible for transhydrogenase-like shunts such as pyruvate carboxylase, malate dehydrogenase, and malic enzyme. The integration of a single gene deletion Ipd1 $\triangle$ and the activation of the transhydrogenase-like shunt further increased isobutanol levels. In a batch fermentation test at the 50-mL scale from $100 \mathrm{~g} / \mathrm{L}$ glucose using the two integrated strains, the isobutanol titer reached $1.62 \pm 0.11 \mathrm{~g} / \mathrm{L}$ and $1.61 \pm 0.03 \mathrm{~g} / \mathrm{L}$ at $24 \mathrm{~h}$ after the start of fermentation, which corresponds to the yield at $0.016 \pm 0.001 \mathrm{~g} / \mathrm{g}$ glucose consumed and $0.016 \pm$ $0.0003 \mathrm{~g} / \mathrm{g}$ glucose consumed, respectively.

Conclusions: These results demonstrate that downregulation of competing pathways and metabolic functions for resolving the cofactor imbalance are promising strategies to construct $S$. cerevisiae strains that effectively produce isobutanol.

Keywords: Isobutanol, Ehrlich pathway, Single-gene deletion, Transhydrogenase-like shunt, Saccharomyces cerevisiae

\section{Background}

There is increasing interest in the production of branched higher alcohols from renewable biomass to be used as a next-generation biofuel and as a building block for commodity chemical production [1,2]. Isobutanol is an important target for biorefinery research because of its preferable properties such as lower toxicity and higher octane values than its straight-chain counterpart [3].

\footnotetext{
* Correspondence: akondo@kobe-u.ac.jp

${ }^{3}$ RIKEN Center for Sustainable Resource Science, 1-7-22 Suehirocho,

Turumi-ku, Yokohama, Kanagawa 230-0045, Japan

${ }^{5}$ Department of Chemical Science and Engineering, Graduate School of Engineering, Kobe University, 1-1 Rokkodaicho, Nada, Kobe 657-8501, Japan Full list of author information is available at the end of the article
}

Metabolically engineered microbial strains to produce isobutanol have been developed by introducing the Ehrlich pathway into bacterial hosts, including Escherichia coli, Corynebacterium glutamicum, Clostridium cellulolyticum, Bacillus subtilis, and cyanobacteria [4-14]. In the recombinant strains, 2-ketoisovalerate, which is an intermediate in the valine biosynthetic pathway, is converted into isobutanol in a two-step reaction: decarboxylation of 2-ketoisovalerate to isobutylaldehyde by 2-keto acid decarboxylase (KDC), and subsequent reduction to isobutanol by alcohol dehydrogenase (ADH) $[4,15]$. In bacterial hosts, isobutanol production near the theoretical maximal yield have been achieved by additional metabolic modifications such as deletion of competing pathways

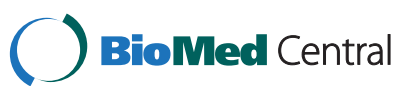


and resolving the cofactor imbalance caused by isobutanol production $[7,8,13]$.

Baker's yeast (Saccharomyces cerevisiae) has advantageous characteristics for cost-effective isobutanol production such as cell-recycling fermentation and tolerance against isobutanol and harsh conditions during fermentation [16]. Isobutanol-producing $S$. cerevisiae strains have been developed following a bacterial strategy by construction of the Ehrlich pathway in the cytosol through expression of the kivd gene from Lactococcus lactis and ADH6 gene from S. cerevisiae [17]. Isobutanol production was increased by the additional activation of the innate valine biosynthetic pathway in mitochondria and by the overexpression of Ilv2p, Ilv5p, and Ilv3p in the cytosol to construct the artificial pathway [18-20]. It was recently reported that the construction of the Ehrlich pathway in mitochondria is effective to increase isobutanol production because of the compartmentalization of the isobutanol biosynthetic pathway [21]. However, the isobutanol yields and titers attained by the developed strains need to

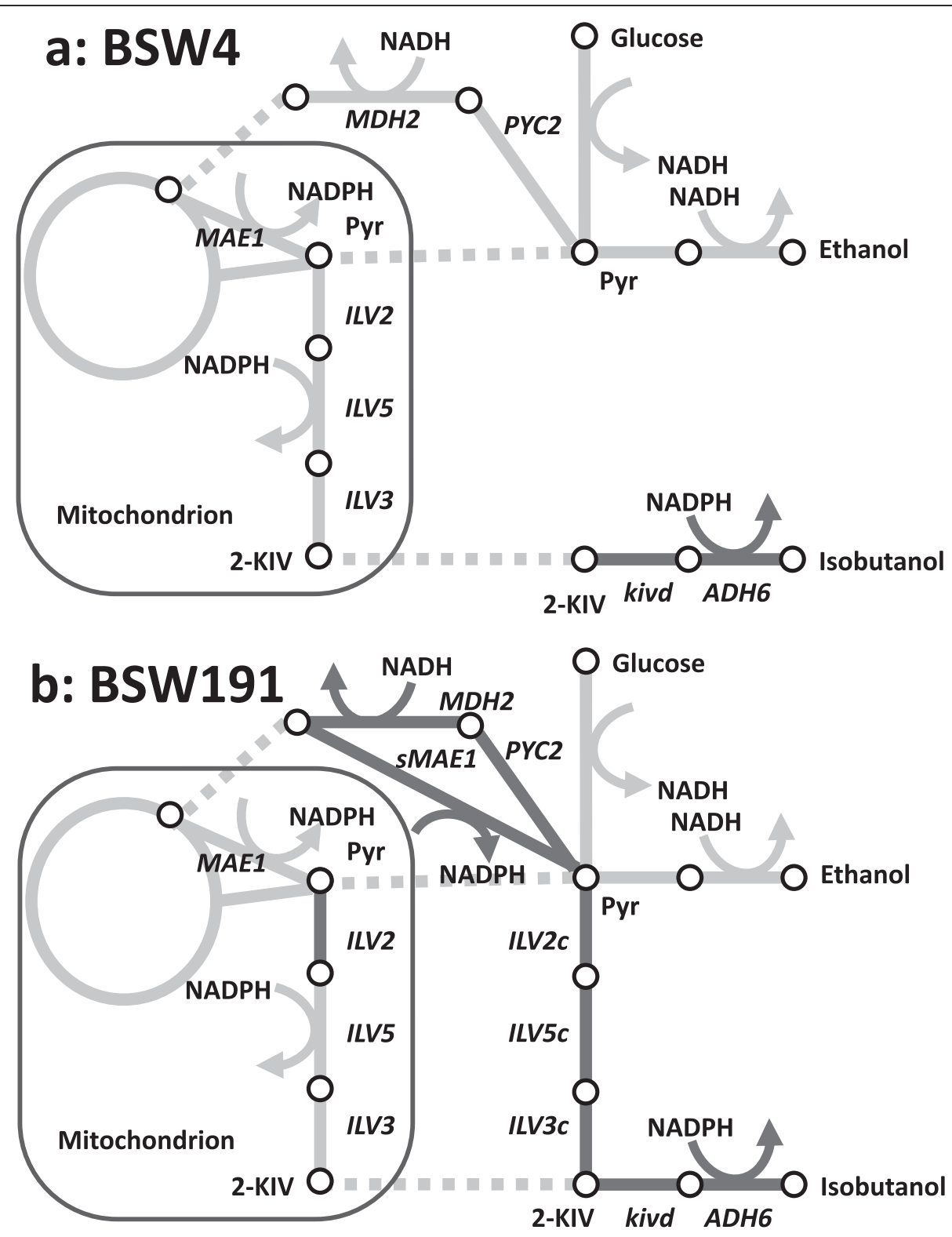

Figure 1 Metabolic maps of the metabolically engineered Saccharomyces cerevisiae strains constructed in this study.

(a) BSW4 strain expressing two genes related to the Ehrlich pathway (kivd and ADH6). (b) BSW191 strain expressing genes related to the Ehrlich pathway (kivd and ADH6), mitochondrial valine biosynthetic pathway (ILV2), artificial valine biosynthetic pathway in the cytosol (ILV2C, ILV5C, and ILV3C), and transhydrogenase-like shunt (PYC2, MDH2, and SMAE1). Black lines indicate the overexpressed or constructed reactions in these strains. Dotted lines represent translocation between mitochondria and cytosol. Pyr, pyruvate; 2-KIV, 2-ketoisovalerate. 
be further improved through engineering of $S$. cerevisiae metabolism. In this study, two strategies including eliminating competing pathways and resolving the cofactor imbalance were applied to improve isobutanol production in $S$. cerevisiae. Isobutanol production was increased by suppressing pyruvate dehydrogenase activity and by activating NADPH regeneration in the cytosol and mitochondria.

\section{Results}

\section{Disruption of genes related to pyruvate metabolism} and valine biosynthesis

The metabolic map shown in Figure 1 indicates that pyruvate is a key intermediate in isobutanol biosynthesis because pyruvate is responsible for several metabolic functions such as the TCA cycle, anaplerotic pathways, and ethanol biosynthesis. Isobutanol production in $S$. cerevisiae was increased by knock-out of the PDC1 gene [17], suggesting that disruption of other genes related to competing pathways should activate isobutanol biosynthesis. In this study, the effects of disruption of pyruvate metabolism-related genes on isobutanol production were examined by constructing single-gene knockout strains (Figure 2a). Three genes required for isobutanol biosynthesis, including ILV2, kivd, and ADH6, were introduced into 12 single-gene knockout strains. The constructed strains were cultivated in $5 \mathrm{~mL}$ of synthetic dextrose (SD) medium under semi-anaerobic conditions. Isobutanol concentration in the medium at $72 \mathrm{~h}$ after the start of cultivation was determined by using gas chromatography-mass spectrometry (GC-MS). The control strain (BSW100, Table 1) was constructed by introducing pGK423-kivd, pGK425-ILV2, and pGK426-ADH6 plasmids (Table 2) into the wild-type strain (BY4741) whose isobutanol production level was $22 \pm 1 \mathrm{mg} / \mathrm{L}$ (Figure 2b).

The fermentation results indicated that the five singlegene deleted strains, including BSW100 irc15 $\Delta$, BSW100
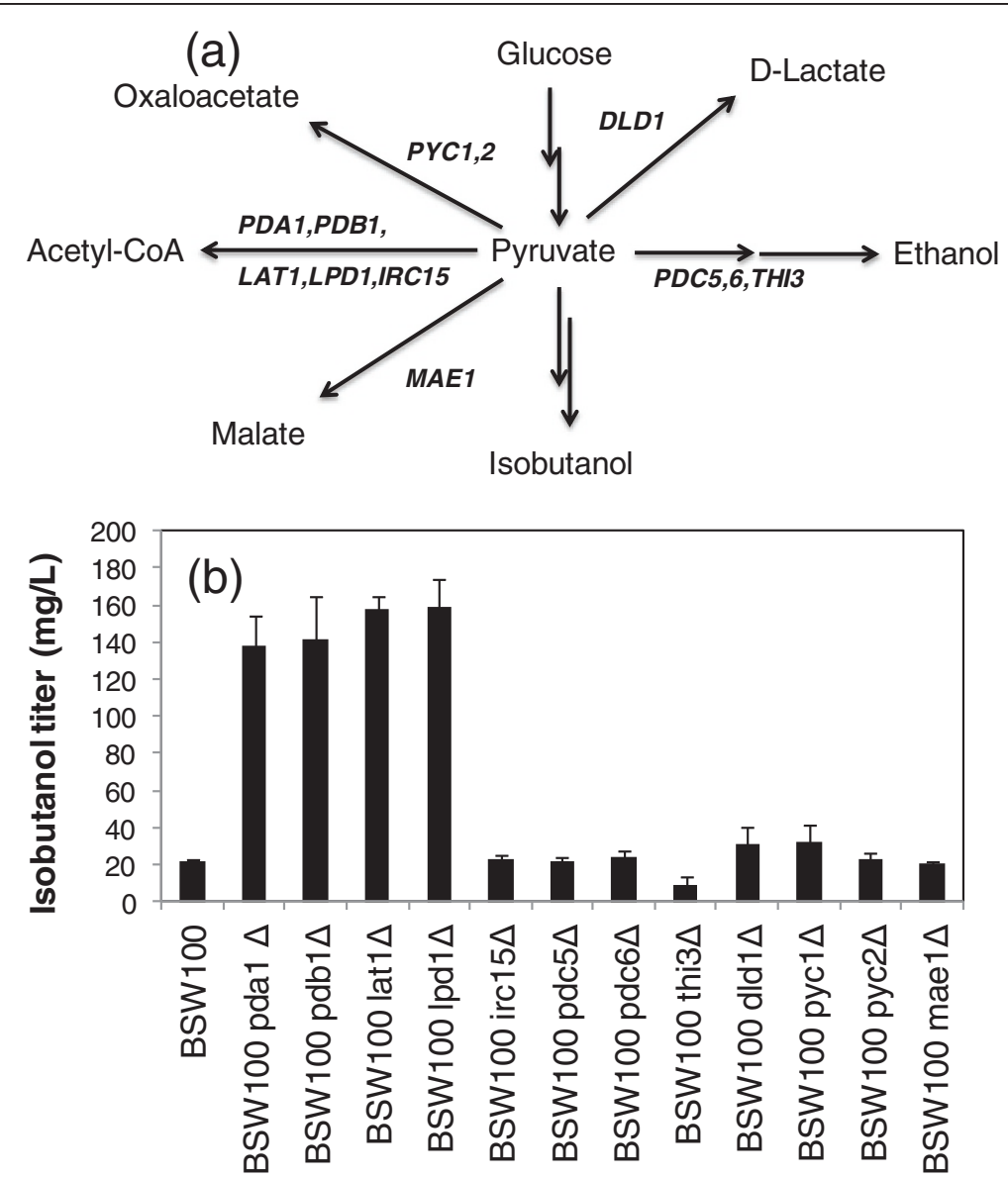

Figure 2 Disruption of genes related to pyruvate metabolism. (a) Genes investigated in this study. (b) Isobutanol production by single gene-deleted strains. All strains including the control strain (BSW100) were constructed by introducing pGK423-kivd, pGK425-ILV2, and pGK426-ADH6 plasmids. Isobutanol titers were determined at $72 \mathrm{~h}$ after the fermentation start. Each data point represents the mean (SD) values obtained from 3 replicate fermentations. 
Table 1 Yeast strains constructed in this study

\begin{tabular}{|c|c|}
\hline Strains & Genotypes \\
\hline $\begin{array}{l}\mathrm{YPH} 499 \\
\end{array}$ & $\begin{array}{l}\text { MATa ura3-52 lys2-801 ade2-101 trp1- } \triangle 63 \text { his3- } \triangle 200 \\
\text { leu2- } \triangle 1\end{array}$ \\
\hline BY4741 & MATa his $3 \triangle 1$ leu $2 \triangle 0$ met $15 \triangle 0$ ura $3 \triangle 0$ \\
\hline BSW100 & BY4741 /pGK423-kivd/pGK425-ILV2/pGK426-ADH6 \\
\hline BSW100 pda1 $\triangle$ & BY4741 pda1 $\triangle$ ppGK423-kivd/pGK425-ILV2/pGK426-ADH6 \\
\hline BSW100 pdb1 $\triangle$ & BY4741 pdb1 $\triangle / p G K 423-k i v d / p G K 425-I L V 2 / p G K 426-A D H 6$ \\
\hline BSW100 lat1 $\triangle$ & BY4741 lat1 $\triangle$ pgGK423-kivd/pGK425-ILV2/pGK426-ADH6 \\
\hline BSW100 Ipd1 $\triangle$ & BY4741 Ipd1 $\triangle$ /pGK423-kivd/pGK425-ILV2/pGK426-ADH6 \\
\hline BSW100 irc15 $\triangle$ & BY4741 irc15 1 pGK423-kivd/pGK425-ILV2/pGK426-ADH6 \\
\hline BSW100 pdc5 $\triangle$ & BY4741 pdc5 $\triangle$ /pGK423-kivd/pGK425-ILV2/pGK426-ADH6 \\
\hline BSW100 pdc6 $\triangle$ & BY4741 pdc6 $\triangle$ /pGK423-kivd/pGK425-ILV2/pGK426-ADH6 \\
\hline BSW100 thi3 $\triangle$ & BY4741 thi3 $\triangle$ /pGK423-kivd/pGK425-ILV2/pGK426-ADH6 \\
\hline BSW100 dld1 $\triangle$ & BY4741 dld1 $\triangle$ /pGK423-kivd/pGK425-ILV2/pGK426-ADH6 \\
\hline BSW100 pyc1 $\triangle$ & BY4741 pyc1 $\triangle / p G K 423-k i v d / p G K 425-I L V 2 / p G K 426-A D H 6$ \\
\hline BSW100 pyc2 $\triangle$ & BY4741 pyc2 $2 / p G K 423-k i v d / p G K 425-I L V 2 / p G K 426-A D H 6$ \\
\hline BSW100 mae1 $\triangle$ & BY4741 mae1 $\triangle$ ppGK423-kivd/pGK425-ILV2/pGK426-ADH6 \\
\hline BSW101 & BY4741/pGK423/pGK425/pGK426 \\
\hline BSW101 pda1 $\triangle$ & BY4741 pda1 $\triangle / p G K 423 / p G K 425 / p G K 426$ \\
\hline BSW101 pdb1 $\triangle$ & BY4741 pdb1 $\triangle / p G K 423 / p G K 425 / p G K 426$ \\
\hline BSW101 lat1 $\triangle$ & BY4741 lat1 $\triangle / p G K 423 / p G K 425 / p G K 426$ \\
\hline BSW101 Ipd1 $\triangle$ & BY4741 lpd1 $\triangle$ /pGK423/pGK425/pGK426 \\
\hline BSW4 & YPH499/pATP426-kivd-ADH6/pATP423 \\
\hline BSW5 & YPH499/pATP426-kivd-ADH6/pATP423-sMAE1 \\
\hline BSW6 & YPH499/pATP426-kivd-ADH6/pATP423-MsM \\
\hline BSW7 & YPH499/pATP426-kivd-ADH6/pATP423-PMsM \\
\hline BSW8 & YPH499/pATP426-kivd-ADH6/pATP423-MAE1 \\
\hline BSW9 & YPH499/pATP426-kivd-ADH6/pATP423-MM \\
\hline BSW10 & YPH499/pATP426-kivd-ADH6/pATP423-PMM \\
\hline BSW13 & YPH499/pATP426-kivd-ADH6/pATP425/pATP423 \\
\hline BSW14 & YPH499/pATP426-kivd-ADH6/pILV2L/pATP423 \\
\hline BSW15 & YPH499/pATP426-kivd-ADH6/pILV2L/pATP423-sMAE1 \\
\hline BSW16 & YPH499/pATP426-kivd-ADH6/pILV2L/pATP423-MsM \\
\hline BSW17 & YPH499/pATP426-kivd-ADH6/pILV2L/pATP423-PMsM \\
\hline BSW18 & YPH499/pATP426-kivd-ADH6/pILV2L/pATP423-MAE1 \\
\hline BSW19 & YPH499/pATP426-kivd-ADH6/pILV2L/pATP423-MM \\
\hline BSW20 & YPH499/pATP426-kivd-ADH6/pILV2L/pATP423-PMM \\
\hline BSW187 & BY4741/pATP426-kivd-ADH6-ILV2/pILV532cytM \\
\hline BSW191 & $\begin{array}{l}\text { BY4741/pATP426-kivd-ADH6-ILV2/pILV532cytM/ } \\
\text { pATP423-PMsM }\end{array}$ \\
\hline BSW192 & $\begin{array}{l}\text { BY4741/pATP426-kivd-ADH6-ILV2/pILV532cytM/ } \\
\text { pATP423-MAE1 }\end{array}$ \\
\hline BSW205 & $\begin{array}{l}\text { BY4741 Ipd1 } \triangle \text { /pATP426-kivd-ADH6-ILV2/pILV532cytM/ } \\
\text { pATP423-MAE1 }\end{array}$ \\
\hline BSW206 & $\begin{array}{l}\text { BY4741 Ipd1 } \triangle \text { /pATP426-kivd-ADH6-ILV2/plLV532cytM/ } \\
\text { pATP423-PMsM }\end{array}$ \\
\hline
\end{tabular}

$\operatorname{pdc5} \Delta$, BSW100 pdc6 $\Delta$, BSW100 mae1 $\Delta$, and BSW100 pyc $2 \Delta$ and had no positive or negative effects on isobutanol production. Among the genes tested, the BSW100 thi3 $\Delta$ strain showed slightly reduced production of isobutanol (Figure 2b). Although this finding suggests that 2-ketoisovalerate may be a substrate of the decarboxylation reaction catalyzed by Thi3p, further characterization of Thi3p is essential for more detailed functional annotation. In contrast, isobutanol production levels were slightly increased in BSW100 dld1 $\Delta$ and BSW100 pyc1 $\Delta$ strains. Furthermore, isobutanol production was remarkably increased to $138-159 \mathrm{mg} / \mathrm{L}$ in BSW100 pda1 $\Delta$, BSW100 pdb1 $\Delta$, BSW100 lpd1 $\Delta$, and BSW100 lat1 $\Delta$ strains (Figure 2b). The cell growth of these mutants was essentially same levels with that of wild type (data not shown). In order to estimate effects of these mutations alone on the isobutanol production, BSW101, BSW101 pda1 $\Delta$, BSW101 pdb1 $\Delta$, BSW101 $\operatorname{lpd} 1 \Delta$, and BSW101 lat1 $\Delta$ strains were constructed by introducing the blank vectors (Table 1). The fermentation test demonstrated that isobutanol production levels were increased to $68-77 \mathrm{mg} / \mathrm{L}$ at $72 \mathrm{~h}$ after the fermentation start for the mutant strains (data not shown). Because the PDA1, PDB1, LPD1, and LAT1 genes encode proteins in the pyruvate dehydrogenase complex, the results indicate that pyruvate supply for isobutanol biosynthesis is increased by reducing the activity of acetyl-CoA biosynthesis in the mitochondria.

\section{Implementation of transhydrogenase-like shunt}

The metabolic pathway shown in Figure 1 also indicates that isobutanol biosynthesis requires NADPH as a cofactor for the reaction catalyzed by Ilv5p and Adh6p. For synthesis of one molecule of isobutanol from two molecules of pyruvate, reducing power has to be supplied by two molecules of NADPH. Because ethanol synthesis from pyruvate essentially uses $\mathrm{NADH}$ as a cofactor, the activation of isobutanol biosynthesis should cause NADPH shortage and NADH abundance. This cofactor imbalance could be relieved by the activity of pyridine nucleotide transhydrogenase catalyzing the following reaction: $\mathrm{NADH}+\mathrm{NADP}^{+} \rightarrow \mathrm{NAD}^{+}+\mathrm{NADPH}[22,23]$. Although pyridine nucleotide transhydrogenase plays an important role in regulating the cellular redox state in many organisms, S. cerevisiae does not possess a gene encoding this enzyme [24]. Furthermore, heterologous expression of a bacterial transhydrogenase was not successful in S. cerevisiae [25].

Recently, it has been demonstrated that the metabolic shunt involving anaplerotic reactions functions similar to transhydrogenase $[3,26,27]$. Through this shunt, pyruvate is sequentially converted to oxaloacetate, malate, and pyruvate by the activity of pyruvate carboxylase (PYC), malate dehydrogenase $(\mathrm{MDH})$, and malic enzyme (MAE), as shown in Figure 1. The net stoichiometry of the shunt is as 
Table 2 Plasmids used in this study

\begin{tabular}{|c|c|c|}
\hline Plasmid & Description & Source or reference \\
\hline pGK423 & $\begin{array}{l}\text { Yeast expression vector containing PGK1 promoter, } 2 \mu \text { origin, HIS3 marker, } \\
\text { no expression (control plasmid) }\end{array}$ & Ishii et al., 2009 [34] \\
\hline pGK425 & $\begin{array}{l}\text { Yeast expression vector containing PGK1 promoter, } 2 \mu \text { origin, LEU2 marker, } \\
\text { no expression (control plasmid) }\end{array}$ & Ishii et al., 2009 [34] \\
\hline pGK426 & $\begin{array}{l}\text { Yeast expression vector containing PGK1 promoter, } 2 \mu \text { origin, URA3 marker, } \\
\text { no expression (control plasmid) }\end{array}$ & Ishii et al., 2009 [34] \\
\hline pATP423 & $\begin{array}{l}\text { Yeast three gene expression vector containing } A D H 1, T D H 3 \text { and } P G K 1 \text { promoters, } \\
2 \mu \text { origin, HIS3 marker, no expression (control plasmid) }\end{array}$ & Ishii et al., in submission \\
\hline pATP425 & $\begin{array}{l}\text { Yeast three gene expression vector containing } A D H 1, T D H 3 \text {, and } P G K 1 \text { promoters, } \\
2 \mu \text { origin, } L E U 2 \text { marker, no expression (control plasmid) }\end{array}$ & Ishii et al., in submission \\
\hline pATP426 & $\begin{array}{l}\text { Yeast three gene expression vector containing } A D H 1, T D H 3 \text {, and } P G K 1 \text { promoters, } \\
2 \mu \text { origin, URA3 marker, no expression (control plasmid) }\end{array}$ & Ishii et al., in submission \\
\hline pGK423-kivd & pGK423, expression of L. lactis 2-ketoisovalerate decarboxylase (kivd) gene & Kondo et al., 2012 [17] \\
\hline pGK425-ILV2 & pGK425, expression of S. cerevisiae ILV2 gene & Kondo et al., 2012 [17] \\
\hline pGK426-ADH6 & pGK426, expression of S. cerevisiae ADH6 gene & Kondo et al., 2012 [17] \\
\hline plLV532cytL & pATP425, co-expression of $S$. cerevisiae ILV5C, ILV3C, and ILV2C genes & This study \\
\hline plLV532cytM & $\begin{array}{l}2 \mu \text { origin, MET15 marker (pGK421-base), co-expression of S. cerevisiae ILV5C, } \\
\text { ILV3C, and ILV2C genes }\end{array}$ & This study \\
\hline PATP423-sMAE1 & PATP423, expression of S. cerevisiae sMAE1 gene & This study \\
\hline PATP423-MsM & pATP423, co-expression of $S$. cerevisiae sMAE1 and MDH2 genes & This study \\
\hline pATP423-PMsM & pATP423, co-expression of S. cerevisiae sMAE1, MDH2, and PYC2 genes & This study \\
\hline pATP423-MAE1 & pATP423, expression of S. cerevisiae MAE1 gene & This study \\
\hline PATP423-MM & pATP423, co-expression of $S$. cerevisiae MAE1 and MDH2 genes & This study \\
\hline PATP423-PMM & pATP423, co-expression of S. cerevisiae MAE1, MDH2, and PYC2 genes & This study \\
\hline pILV2L & pATP425, expression of S. cerevisiae ILV2 gene & This study \\
\hline pATP426-kivd-ADH6 & pATP426, co-expression of L. lactis kivd and S. cerevisiae ADH6 genes & This study \\
\hline pATP426-kivd-ADH6-ILV2 & pATP426, co-expression of L. lactis kivd, S. cerevisiae ADH6, and ILV2 genes & This study \\
\hline
\end{tabular}

follows: $\mathrm{ATP}+\mathrm{NADH}+\mathrm{NADP}^{+} \rightarrow \mathrm{ADP}+\mathrm{Pi}+\mathrm{NAD}^{+}+$ $\mathrm{NADPH}$, because the coenzyme preferences of $\mathrm{MDH}$ and MAE in S. cerevisiae are NADH and NADP+, respectively [28]. The transhydrogenase-like shunt successfully resolved the cofactor imbalance in xylose-fermenting yeast expressing xylose reductase and xylulose dehydrogenase [27].

In this study, two versions of transhydrogenase-like shuts were implemented to improve isobutanol production by distinct localization of malic enzyme (Mae1p), which is originally expressed in the mitochondria [29]. In the first version, Mae1p activity in the mitochondria was overexpressed to supply NADPH to the valine biosynthesis pathway. In the second version, NADPH was supplied to the Ehrlich pathway by the cytosolic expression of Mae1p (Figure 1b). For this, two plasmids, pATP423-MAE1 and pATP423-sMAE1, were constructed as shown in Figure 3a. The native $M A E 1$ gene and its truncated fragment ( $S M A E 1$ ) lacking the sequence of the mitochondrial transit signal [26] was inserted into an open reading frame (ORF) of the pATP423 plasmid. Plasmids additionally harboring $M D H 2$ and $P Y C 2$ genes are also constructed by introducing the other ORFs of pATP423 plasmids (pATP423-MsM, pATP423-PMsM, pATP423MM, and pATP423-PMM, Figure $3 b$ and c). Mdh2p and Pyc2p are localized to the cytosol [27] (Figure 1).

The pATP423-sMAE1, pATP423-MsM, and pATP423PMsM plasmids were introduced into the YPH499 pATP426-kivd-ADH6 strain to construct a transhydro genase-like shunt in the cytosol. There is a significant increase in isobutanol formation in BSW 6 and BSW 7 indicating that there is a slight activation of isobutanol production by implementation of the transhydrogenaselike shunt (Figure 4a). Furthermore, the BSW8 strain overexpressing mitochondrial MAE1p through introduction of the pATP423-MAE1 plasmid showed a 1.6-fold increase in isobutanol titer $(71 \pm 6 \mathrm{mg} / \mathrm{L})$ at $48 \mathrm{~h}$ after the start of fermentation. Isobutanol production was activated by the additional overexpression of Mdh2p and Pyc2p in the BSW10 strain, and the isobutanol titer reached $83 \pm 2 \mathrm{mg} / \mathrm{L}$ (Figure $4 \mathrm{a}$ ).

The transhydrogenase-like shuts were also introduced for the strain whose isobutanol biosynthetic pathway in 

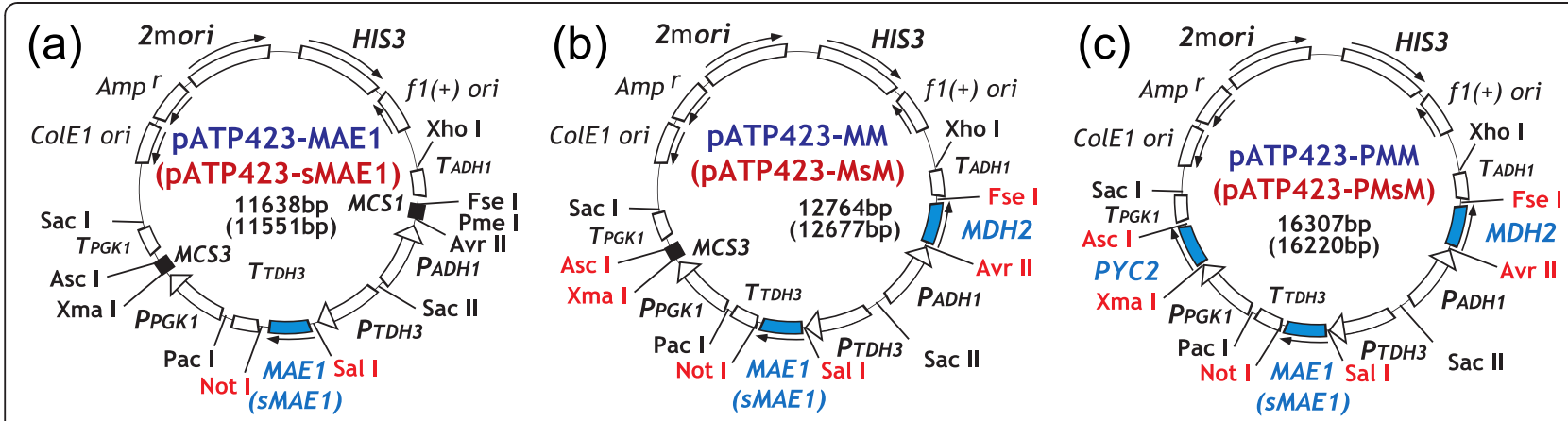

Figure 3 Plasmids for co-expression of genes related to the transhydrogenase-like shunt. (a) pATP423-MAE1 and pATP423-sMAE1 for the mitochondrial and cytosolic expressions of MAE1p. SMAE1 is a short form of MAE1 lacking the first 90 nucleotides. (b) pATP423-MM and pATP423-MsM for co-expressions of MDH2p and MAE1p. (c) pATP423-PMM and pATP423-PMsM for co-expressions of PYC2p, MDH2p and MAE1p.

the mitochondria was activated by the overexpression of Ilv2p (Figure 4a). The isobutanol production in the control strain BSW13 (23 \pm 3 mg/L, YPH499/pATP426kivd-ADH6/pATP425/pATP423) is lower than that of BSW4 (45 $\pm 4 \mathrm{mg} / \mathrm{L}$, YPH499/pATP426-kivd-ADH6/ pATP423). Since the leucine biosynthesis is branched from the valine biosynthesis and thus competing with the isobutanol biosynthesis, the leu2- $\Delta$ 1allele in BSW4 strain should have positive effect on isobutanol biosynthesis. On the other hands, the BMW13 strain showed a leucine-autotrophy by an additional introduction of ATP425 encoding LEU2 gene. The isobutanol level was increased to $117 \pm 6 \mathrm{mg} / \mathrm{L}$ by the overexpression of Mdh2p, Pyc2p, and cytosolic sMae1p (BSW17 strain, Figure 4a), probably because of the simultaneous upregulation of 2-ketoisovalerate and NADPH supply in cytosol for isobutanol synthesis via the Ehrlich pathway. Furthermore, activation of the NADPH supply in the mitochondria by overexpression of mitochondrial Mae1p also increased the isobutanol titer to $139 \pm 4 \mathrm{mg} / \mathrm{L}$, as shown for the BSW18 strain. The isobutanol yields of BSW17 and BSW18 strains were $0.006 \pm 0.0003$ and $0.007 \pm 0.0002 \mathrm{~g} / \mathrm{g}$ glucose consumed, respectively, since glucose was completely consumed at $48 \mathrm{~h}$ after the fermentation start (Figure $4 \mathrm{~b}$ ). The fermentation profiles indicated that the glucose consumption rates and the ethanol production rate of BSW17 and BSW18 were essentially identical with that of BSW13 (Figure 4b). The isobutanol titer was decreased, however, by additional introduction of the $\mathrm{MDH} 2$ and $\mathrm{PYC} 2$ genes (BSW19 and BSW20 strains), which suggests that there is an optimal balance of enzyme activities among pyruvate carboxylase, malate dehydrogenase, and malic enzyme.

\section{Construction of isobutanol overproducing strains}

Metabolically engineered strains overproducing isobutanol were constructed by integrating the gene disruption and the transhydrogenase-like shunts examined in this study. Isobutanol production by the BSW187 strain (BY4741/pATP426-kivd-ADH6-ILV2/pILV532cytM) possessing genes for the Ehrlich pathway, activation of the mitochondrial valine biosynthetic pathway (ILV2), and the artificial pathway for 2-ketoisovalerate biosynthesis in the cytosol was $46 \pm 14 \mathrm{mg} / \mathrm{L}$ (Figure 5). The cytosolic artificial pathway was constructed by the expression of truncated $I L V 2 c, I L V 3 c$, and $I L V 5 c$ genes in which the mitochondrial translocation signals of the ILV2, ILV3, and ILVS genes were deleted. The details of the artificial pathway were described in our previous study [20]. It should be noted that the isobutanol production by the metabolically engineered strains with the BY4741 background were significantly lower than that by strains with the YPH499 background; the reason for this difference is unclear $[17,20]$.

The isobutanol titer was increased by the additional introduction of transhydrogenase-like shunts as shown in BSW192 and BSW191 strains possessing pATP423MAE1 and pATP423-PMsM plasmids, respectively. The isobutanol titer was increased to $94 \pm 5$ and $83 \pm 2 \mathrm{mg} / \mathrm{L}$ by the activation of the NADPH supply in the cytosol and mitochondria, respectively. The cell growth was not affected by introducing pATP423-MAE1 and pATP423PMsM plasmids (data not shown). The additional disruption of the LPD1 gene in the BSW192 and BSW191 strains further activated isobutanol biosynthesis. The isobutanol titer of the BSW205 and BSW206 strains reached $230 \pm 13$ and $221 \pm 27 \mathrm{mg} / \mathrm{L}$, respectively (Figure 5) that correspond to isobutanol yields at $0.012 \pm 0.0007$ and $0.011 \pm 0.001 \mathrm{~g} / \mathrm{g}$ glucose consumed, respectively. The glucose were completely consumed at $48 \mathrm{~h}$ after the fermentation start (data not shown).

The fermentation profile of the BSW205 and BSW206 strains was determined by batch fermentation at a $50-\mathrm{mL}$ scale under semi-anaerobic conditions. The yeast cells were inoculated in $50 \mathrm{~mL}$ of $\mathrm{SD}$ medium containing $100 \mathrm{~g} / \mathrm{L}$ glucose. As shown in Figure 6, the isobutanol titer reached $1.62 \pm 0.11$ and $1.61 \pm 0.03 \mathrm{~g} / \mathrm{L}$ at $24 \mathrm{~h}$ after the start of fermentation, which corresponded to isobutanol yields of $0.016 \pm 0.001 \mathrm{~g} / \mathrm{g}$ glucose consumed and $0.016 \pm$ $0.0003 \mathrm{~g} / \mathrm{g}$ glucose consumed, respectively. The fermentation 

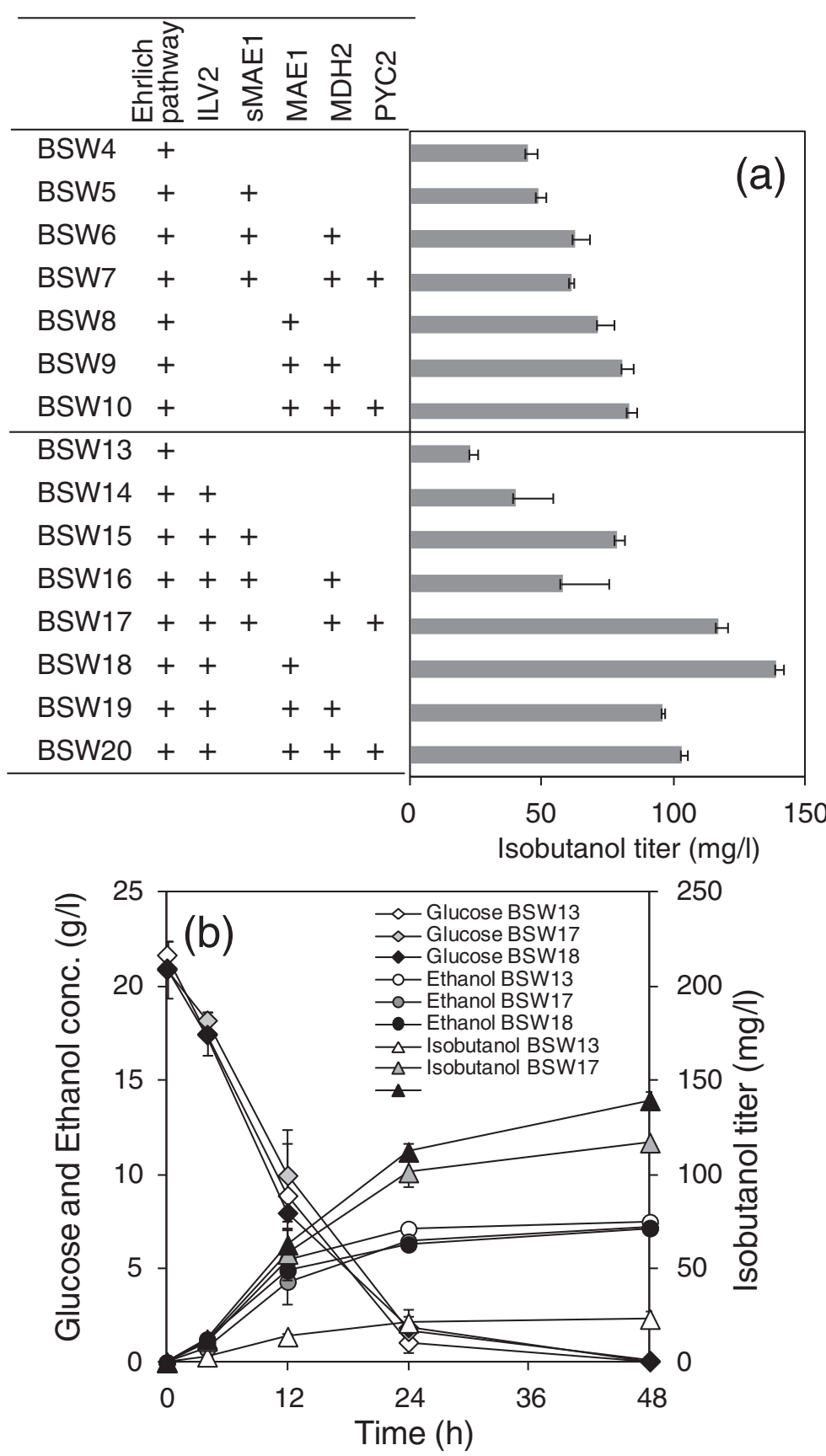

Figure 4 Isobutanol production by transformants expressing genes related to the transhydrogenase-like shunt. (a) Isobutanol production by S. cerevisiae strains co-expressing genes related to the Ehrlich pathway (kivd and ADH6), the ILV2 gene, and transhydrogenase-like shunt. Isobutanol titers were determined at $48 \mathrm{~h}$ after the fermentation start. The introduced pathway and genes are shown in the figures. (b) Fermentation profiles of BSW13, BSW17 and BSW18. Detailed genotypes of each strain are described in Table 1. Each data point represents the mean (SD) values obtained from 3 replicate fermentations.

test also demonstrated that the BSW205 and BSW206 strains actively consumed and produced glucose and ethanol, respectively. The ethanol yield of the BSW205 and BSW206 strains was $0.42 \pm 0.01$ and $0.42 \pm 0.003 \mathrm{~g} / \mathrm{g}$ glucose consumed, respectively.

\section{Discussion}

In the metabolic engineering of microbial cell factories, elimination of competing pathways and resolution of the cofactor imbalance are essential to improve production of target compounds, as has been demonstrated in the 


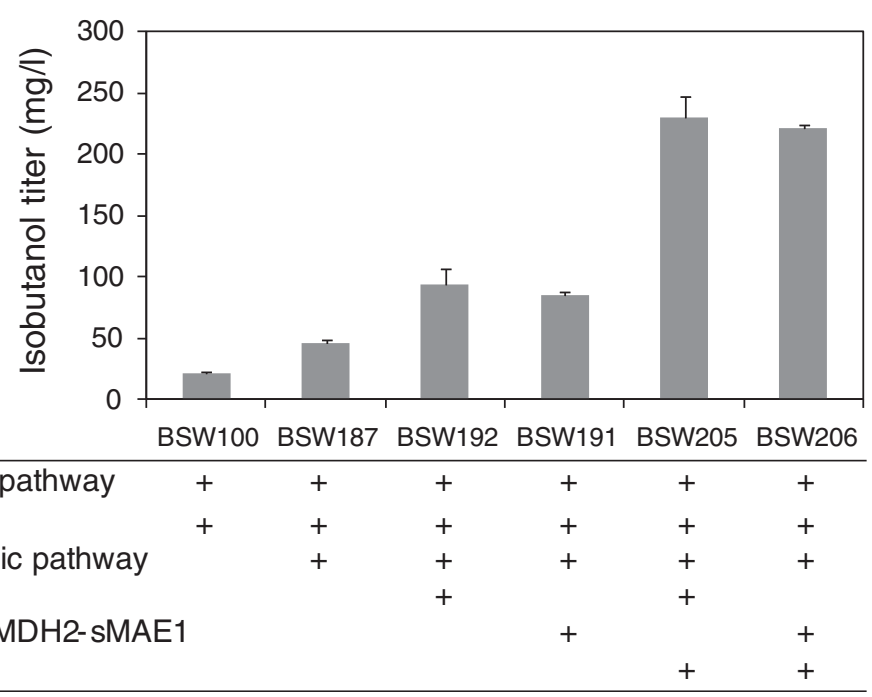

Figure 5 Isobutanol production by metabolically engineered S. cerevisiae transformants. These transformants are co-expressing genes related to the Ehrlich pathway (kivd and ADH6), activation of the mitochondrial valine biosynthetic pathway (ILV2), the artificial pathway for 2-ketoisovalerate biosynthesis in the cytosol (Cytosolic pathway) and two versions of transhydrogenase-like shunt (MAE1 and PYC2-MDH2-sMAE1) in combination with the single-gene deletion of LPD1 (IpdI $\triangle$ ). Isobutanol titers were determined at $48 \mathrm{~h}$ after the fermentation start. Each data point represents the mean (SD) values obtained from 3 replicate fermentations.

construction of recombinant E. coli and C. glutamicum overproducing isobutanol $[3,4,13]$. In this study, these strategies were applied to increase isobutanol production in S. cerevisiae. The metabolic simulation of single genedeletion strains demonstrated that suppression of ethanol biosynthesis by the deletion of alcohol dehydrogenase genes is effective to increase isobutanol yield [28]. Although a recombinant $S$. cerevisiae strain lacking five

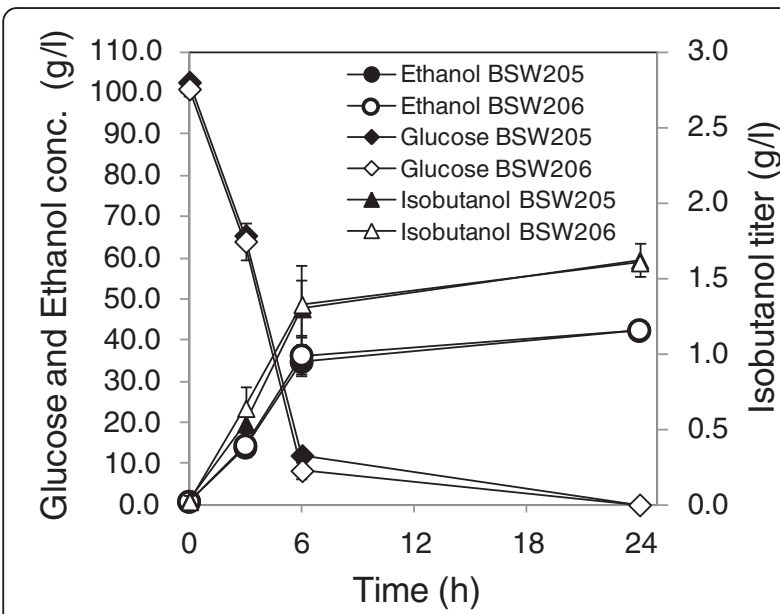

Figure 6 Fermentation profiles of the BSW205 and BSW206 strains by batch fermentations at $50-\mathrm{mL}$ scale under semi-anaerobic conditions. The yeast cells were inoculated in $50 \mathrm{~mL}$ of SD medium containing $100 \mathrm{~g} / \mathrm{L}$ glucose. Closed and open symbols represent data of BSW205 and BSW206, respectively. Circles, Diamonds, and Triangles represent the titers of ethanol, glucose, and isobutanol, respectively. Each data point represents the mean (SD) values obtained from 3 replicate fermentations. alcohol dehydrogenase genes has recently been constructed [30], this strategy is unlikely to be effective because the Ehrlich pathway for isobutanol biosynthesis also requires an alcohol dehydrogenase gene (ADH6). Thus, to find other gene deletion targets, the isobutanol production of 12 single gene-deletion mutants expressing genes for isobutanol biosynthesis was experimentally investigated (Figure 2). A fermentation test showed that isobutanol production was significantly increased in strains lacking genes responsible for the pyruvate dehydrogenase (PDH) complex. In these strains, PDH activity was reduced but not eliminated by the deletion of a single protein of the complex, which resulted in increased carbon flux into isobutanol biosynthesis. These results highlight mechanisms regulating isobutanol biosynthesis in $S$. cerevisiae that could not be identified by computer simulation of metabolism based on metabolic flux balance analysis.

In the second part of this study, the cofactor imbalance in isobutanol-producing S. cerevisiae strains was resolved by introducing transhydrogenase-like shunts (Figure 4 and 5). The transhydrogenase-like shunt consisted of Pyc2p, Mdh2p, and Mae1p originally existing in S. cerevisiae (Figure 1) [26,31]. However, the shunt was hardly functional in S. cerevisiae because the deletion of these genes showed no negative effect on isobutanol production (Figure 2). The recombinant strains overexpressing Mae1p showed improved production of isobutanol (Figure 4a), which indicates that increased NADPH and pyruvate supply in mitochondria through the activation of mitochondrial malic enzyme (Maelp) could be a driving force to increase isobutanol biosynthesis. It was 
also demonstrated that isobutanol production in S. cerevisae was increased by the expression of malic enzyme in the cytosol (sMae1p) to supply NADPH in the cytosol (Figure 4a). The effect of the transhydrogenase-like function on $\mathrm{NADH} / \mathrm{NAD}^{+}$and $\mathrm{NADPH} / \mathrm{NADP}^{+}$levels was confirmed in xylose-fermenting $S$. cerevisae overexpressing the shunt [27]. The improvement of isobutanol production in S. cerevisiae by resolving the cofactor imbalance was demonstrated for the first time in this study.

The integration of PDH suppression by $l p d 1 \Delta$ and activation of the transhydrogenase-like shunt in BSW205 and BSW206 strains successfully increased the isobutanol levels to $230 \pm 13$ and $221 \pm 27 \mathrm{mg} / \mathrm{L}$, respectively (Figure 5). In the batch fermentation test at the $50-\mathrm{mL}$ scale from $100 \mathrm{~g} / \mathrm{L}$ glucose using these recombinant strains, the isobutanol titer reached $1.62 \pm 0.11$ and $1.61 \pm$ $0.03 \mathrm{~g} / \mathrm{L}$ at $24 \mathrm{~h}$ after the start of fermentation (Figure 6). The titer corresponds to the yield at $0.016 \pm 0.001$ and $0.016 \pm 0.0003 \mathrm{~g} / \mathrm{g}$ glucose consumed, respectively.

\section{Conclusions}

All recombinant strains constructed in this study chiefly produced ethanol. For instance, the ethanol yield from glucose of BSW205 and BSW206 strains in the batch fermentation test at the $50-\mathrm{mL}$ scale was $0.42 \pm 0.01$ and $0.42 \pm 0.003 \mathrm{~g} / \mathrm{g}$ glucose consumed, respectively (Figure 6), which indicates that restriction of ethanol biosynthesis from pyruvate is unavoidable to drastically improve isobutanol yield. However, it has been demonstrated that downregulation of ethanol biosynthesis by deleting the pyruvate decarboxylase and alcohol dehydrogenase genes seriously hampers active cell growth [30,32]. In this regard, identification of the $M T H 1 \triangle T$ allele is a promising because the mutation enables $S$. cerevisiae strains lacking three pyruvate dehydrogenase genes to actively grow with reduced ethanol production [33]. Recombinant $S$. cerevisiae strains effectively producing isobutanol will be constructed by integrating further activation of pathways for isobutanol biosynthesis [19-21], the down-regulation of competing pathways for acetylCoA and ethanol synthesis, and metabolic functions for resolving the cofactor imbalance as demonstrated in this study.

\section{Methods}

Strains, plasmids, and yeast transformation

The yeast strains used in this study are listed in Table 1. S. cerevisiae YPH499 (MATa ura3-52 lys2-801 ade2-101 trp1- $\Delta 63$ his3- $\Delta 200$ leu2- $\Delta 1$, purchased from Stratagene, La Jolla, CA, USA) (Sikorski and Hieter, 1989), BY4741

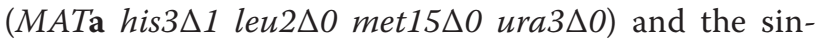
gle gene deletion mutants (purchased from Thermo Scientific) were used for yeast host strains. The plasmids and primers used in this study are summarized in Tables 2 and 3 , respectively. All plasmids were derived from the pGK and pATP vector series, in which gene expression is controlled by the PGK1 promoter and the ADH1, TDH1, and PGK1 promoters, respectively [34] (Ishii et al., in submission). The kivd gene from Lactococcus lactis was synthesized by Invitrogen Life Technologies Corp.

Table 3 Primers used in this study

\begin{tabular}{|c|c|c|}
\hline Target genes & Primers & Restriction enzymes \\
\hline $\operatorname{lLV} 5 \mathrm{C}(\mathrm{fw})$ & 5'-ttttCCTAGGatgttgaagcaaatcaacttcggtggtact & Avrll \\
\hline $\operatorname{lLV} 5 \mathrm{C}(r v)$ & 5'-ttttGGCCGGCCttattggttttctggtctcaactttctg & Fsel \\
\hline $\operatorname{ILV} 3 \mathrm{C}(\mathrm{fw})$ & 5'-aaaaGTCGACatgctttatgccaccggtttcaagaaggaa & Sall \\
\hline $\operatorname{lLV} 3 c(r v)$ & 5'-ggggGCGGCCGCtcaagcatctaaaacacaaccgttggaa & Notl \\
\hline $\operatorname{lLV} 2 \mathrm{C}(\mathrm{fw})$ & 5'-ccccCCCGGGatgccagagcctgctccaagtttcaatgtt & Xmal \\
\hline $\operatorname{lLV} 2 \mathrm{C}(r \mathrm{r})$ & 5'-ttttGGCGCGCCtcagtgcttaccgcctgtacgcttatga & Ascl \\
\hline $\mathrm{MDH} 2$ (fw) & 5'-aaaaCCTAGGatgcctcactcagttacacc & Avrll \\
\hline $\mathrm{MDH} 2(r v)$ & 5'-aaaaGGCCGGCCttaagatgatgcagatctcg & Fsel \\
\hline sMAE1 (fw) & 5'-ttttGTCGACatgtggcctattcagcaatcgcg & Sall \\
\hline sMAE1 (rv) & 5'-ttttGCGGCCGCctacaattggttggtgtgca & Notl \\
\hline MAE1 (fW) & 5'-ccccGTCGACatgcttagaaccagactatc & Sall \\
\hline MAE1 ( $r$ ) & 5’ttttGCGGCCGCctacaattggttggtgtgca & Notl \\
\hline PYC2 (fw) & 5'-aaaaCCCGGGatgagcagtagcaagaaatt & Xmal \\
\hline PYC2 (rv) & 5'-aaaaGGCGCGCCttactttttttgggatgggg & Asc 1 \\
\hline ADH6 (fw) & 5’ggggCCTAGGatgtcttatcctgagaaa & Avrll \\
\hline$A D H 6(r v)$ & 5'-aaaaGGCCGGCCctagtctgaaaattctttgt & Fsel \\
\hline kivd (fw) & 5'-ccccGTCGACatgtatacagtaggagatta & Sall \\
\hline kivd (rv) & 5'-ccccGCGGCCGCttatgatttattttgttcag & Notl \\
\hline
\end{tabular}


(Carlsbad, CA). All other genes derived from S. cerevisiae were amplified from YPH499 genomic DNA using primers shown in Table 3. A short form of MAE1 lacking the first 90 nucleotides ( $S M A E 1)$ was cloned from the YPH499 genome using sMAE1 (fw) and sMAE1 (rv) primers. pILV532cytM was constructed by inserting a XhoI/SacI-digested fragment of pILV532cytL into the same sites of the pGK421 vector harboring the MET15 marker and a $2 \mu$ origin backbone. The growth conditions, DNA techniques, and lithium-acetate method for transformations were previously described $[35,36]$.

\section{Isobutanol fermentation}

The transformants were cultured for $72 \mathrm{~h}$ at $30^{\circ} \mathrm{C}$ in $5 \mathrm{~mL}$ of SD minimal medium $(6.7 \mathrm{~g} / \mathrm{L}$ yeast nitrogen base without amino acids and $20 \mathrm{~g} / \mathrm{L}$ glucose) containing the required amino acids. Following centrifugation at $3,000 \mathrm{rpm}$ for $5 \mathrm{~min}$ and removal of supernatant, the yeast cells were cultured in $5 \mathrm{~mL}$ of fresh SD minimal medium containing the required amino acids. The concentrations of isobutanol and ethanol in the medium at 48 and $72 \mathrm{~h}$ after the start of fermentation were determined using GC-MS (GCMS-QP2010 Plus; Shimadzu) following a previously described procedure [17]. The glucose concentrations were determined by Glucose C-II Test Wako Kit (Wako Pure Chemicals, Tokyo, Japan).

For flask-scale fermentation, the yeast transformants were aerobically cultivated in SD minimal medium containing the required amino acids for $48 \mathrm{~h}$ at $30^{\circ} \mathrm{C}$. The cells were collected by centrifugation at $1,000 \times g$ for $5 \mathrm{~min}$ at $4^{\circ} \mathrm{C}$ and washed twice with sterile water. The cells were then inoculated into $50 \mathrm{~mL}$ of YP medium (containing $10 \mathrm{~g} / \mathrm{L}$ yeast extract and $20 \mathrm{~g} / \mathrm{L}$ peptone) with $100 \mathrm{~g} / \mathrm{L}$ glucose. The initial cell concentration was adjusted to $30 \mathrm{~g}$ of wet cells per liter (corresponding to OD 20 and $6.7 \mathrm{~g}$ of dry cells per liter). All fermentations were performed at $30^{\circ} \mathrm{C}$ with mild agitation in $100-\mathrm{mL}$ closed bottles equipped with a bubbling $\mathrm{CO}_{2}$ outlet.

\section{Competing interests}

We declare that the authors have no conflicts of interest in connection with this paper.

\section{Authors' contributions}

JI, TK, FM, HT, and KI performed the experiments. FM, Jl, and TK analyzed the data. FM, JI, TK, and AK designed the study. JI, FM, and JI wrote the paper. All authors read and approved the final manuscript.

\section{Acknowledgements \\ This work was financed by the Industrial Technology Research Grant Program for 2011 of the New Energy and Industrial Technology Development Organization (NEDO) of Japan and Special Coordination Funds for Promoting Science and Technology, Creation of Innovation Centers for Advanced Interdisciplinary Research Areas (Innovative Bioproduction Kobe), MEXT, Japan. This work is also supported in part by Grants in Aid for Scientific Research (B) No 25820400.}

\section{Author details}

${ }^{1}$ Department of Bioinformatic Engineering, Graduate School of Information Science and Technology, Osaka University, 1-5 Yamadaoka, Suita, Osaka
565-0871, Japan. ${ }^{2}$ Organization of Advanced Science and Technology, Kobe University, 1-1 Rokkodaicho, Nada, Kobe 657-8501, Japan. ${ }^{3}$ RIKEN Center for Sustainable Resource Science, 1-7-22 Suehirocho, Turumi-ku, Yokohama, Kanagawa 230-0045, Japan. ${ }^{4}$ Division of Natural Environment and Information, Faculty of Environment and Information Sciences, Yokohama National University, 79-7, Tokiwadai, Hodogaya, Yokohama 240-8501, Japan. ${ }^{5}$ Department of Chemical Science and Engineering, Graduate School of Engineering, Kobe University, 1-1 Rokkodaicho, Nada, Kobe 657-8501, Japan.

Received: 8 July 2013 Accepted: 5 November 2013

Published: 5 December 2013

\section{References}

1. Weber C, Farwick A, Benisch F, Brat D, Dietz H, Subtil T, Boles E: Trends and challenges in the microbial production of lignocellulosic bioalcohol fuels. Appl Microbiol Biotechnol 2010, 87(4):1303-1315.

2. Connor MR, Liao JC: Microbial production of advanced transportation fuels in non-natural hosts. Curr Opin Biotechnol 2009, 20(3):307-315.

3. Blombach B, Eikmanns BJ: Current knowledge on isobutanol production with Escherichia coli, Bacillus subtilis and Corynebacterium glutamicum. Bioeng Bugs 2011, 2(6):346-350.

4. Atsumi S, Hanai T, Liao JC: Non-fermentative pathways for synthesis of branched-chain higher alcohols as biofuels. Nature 2008, 451(7174):86-89.

5. Atsumi S, Higashide W, Liao JC: Direct photosynthetic recycling of carbon dioxide to isobutyraldehyde. Nat Biotechnol 2009, 27(12):1177-1180.

6. Atsumi S, Li Z, Liao JC: Acetolactate synthase from Bacillus subtilis serves as a 2-ketoisovalerate decarboxylase for isobutanol biosynthesis in Escherichia coli. Appl Environ Microbiol 2009, 75(19):6306-6311.

7. Atsumi S, Wu TY, Eckl EM, Hawkins SD, Buelter T, Liao JC: Engineering the isobutanol biosynthetic pathway in Escherichia coli by comparison of three aldehyde reductase/alcohol dehydrogenase genes. Appl Microbiol Biotechnol 2010, 85(3):651-657.

8. Baez A, Cho KM, Liao JC: High-flux isobutanol production using engineered Escherichia coli: a bioreactor study with in situ product removal. Appl Microbiol Biotechnol 2011, 90(5):1681-1690.

9. Bastian S, Liu X, Meyerowitz JT, Snow CD, Chen MM, Arnold FH: Engineered ketol-acid reductoisomerase and alcohol dehydrogenase enable anaerobic 2-methylpropan-1-ol production at theoretical yield in Escherichia coli. Metab Eng 2011, 13(3):345-352.

10. Blombach B, Riester T, Wieschalka S, Ziert C, Youn JW, Wendisch VF, Eikmanns BJ: Corynebacterium glutamicum tailored for efficient isobutanol production. Appl Environ Microbiol 2011, 77(10):3300-3310.

11. Higashide W, Li Y, Yang Y, Liao JC: Metabolic engineering of Clostridium cellulolyticum for production of isobutanol from cellulose. Appl Environ Microbiol 2011, 77(8):2727-2733.

12. Smith KM, Cho KM, Liao JC: Engineering Corynebacterium glutamicum for isobutanol production. Appl Microbiol Biotechnol 2010, 87(3):1045-1055.

13. Yamamoto S, Suda M, Niimi S, Inui M, Yukawa H: Strain optimization for efficient isobutanol production using Corynebacterium glutamicum under oxygen deprivation. Biotechnol Bioeng 2013, 110(10):2938-2948.

14. Li S, Wen J, Jia X: Engineering Bacillus subtilis for isobutanol production by heterologous Ehrlich pathway construction and the biosynthetic 2-ketoisovalerate precursor pathway overexpression. Appl Microbiol Biotechnol 2011, 91(3):577-589.

15. Hazelwood LA, Daran JM, van Maris AJ, Pronk JT, Dickinson JR: The Ehrlich pathway for fusel alcohol production: a century of research on Saccharomyces cerevisiae metabolism. Appl Environ Microbiol 2008, 74(8):2259-2266.

16. Kondo A, Ishii J, Hara KY, Hasunuma T, Matsuda F: Development of microbial cell factories for bio-refinery through synthetic bioengineering. J Biotechnol 2013, 163(2):204-216.

17. Kondo T, Tezuka H, Ishii J, Matsuda F, Ogino C, Kondo A: Genetic engineering to enhance the Ehrlich pathway and alter carbon flux for increased isobutanol production from glucose by Saccharomyces cerevisiae. Journal of Biotechnology 2012, 159(1-2):32-37.

18. Chen X, Nielsen KF, Borodina I, Kielland-Brandt MC, Karhumaa K: Increased isobutanol production in Saccharomyces cerevisiae by overexpression of genes in valine metabolism. Biotechnol Biofuels 2011, 4:21.

19. Brat D, Weber C, Lorenzen W, Bode HB, Boles E: Cytosolic re-localization and optimization of valine synthesis and catabolism enables inseased isobutanol production with the yeast Saccharomyces cerevisiae. Biotechnol Biofuels 2012, 5(1):65. 
20. Matsuda F, Kondo T, Ida K, Tezuka H, Ishii J, Kondo A: Construction of an artificial pathway for isobutanol biosynthesis in the cytosol of Saccharomyces cerevisiae. Biosci Biotechnol Biochem 2012, 76(11):2139-2141.

21. Avalos JL, Fink GR, Stephanopoulos G: Compartmentalization of metabolic pathways in yeast mitochondria improves the production of branched-chain alcohols. Nat Biotechnol 2013, 31:335-341.

22. Arkblad EL, Betsholtz C, Rydstrom J: The cDNA sequence of proton-pumping nicotinamide nucleotide transhydrogenase from man and mouse. Biochim Biophys Acta 1996, 1273(3):203-205.

23. Sauer U, Canonaco F, Heri S, Perrenoud A, Fischer E: The soluble and membrane-bound transhydrogenases $U d h A$ and $P n t A B$ have divergent functions in NADPH metabolism of Escherichia coli. J Biol Chem 2004, 279(8):6613-6619.

24. Anderlund M, Nissen TL, Nielsen J, Villadsen J, Rydstrom J, Hahn-Hagerdal B, Kielland-Brandt MC: Expression of the Escherichia coli pntA and pntB genes, encoding nicotinamide nucleotide transhydrogenase, in Saccharomyces cerevisiae and its effect on product formation during anaerobic glucose fermentation. Appl Environ Microbiol 1999, 65(6):2333-2340.

25. Nissen TL, Anderlund M, Nielsen J, Villadsen J, Kielland-Brandt MC: Expression of a cytoplasmic transhydrogenase in Saccharomyces cerevisiae results in formation of 2-oxoglutarate due to depletion of the NADPH pool. Yeast 2001, 18(1):19-32.

26. Moreira dos Santos M, Raghevendran V, Kotter P, Olsson L, Nielsen J: Manipulation of malic enzyme in Saccharomyces cerevisiae for increasing NADPH production capacity aerobically in different cellular compartments. Metab Eng 2004, 6(4):352-363.

27. Suga H, Matsuda F, Hasunuma T, Ishii J, Kondo A: Implementation of a transhydrogenase-like shunt to counter redox imbalance during xylose fermentation in Saccharomyces cerevisiae. Appl Microbiol Biotechnol 2012, 97(4):1669-1678.

28. Matsuda F, Furusawa C, Kondo T, Ishii J, Shimizu H, Kondo A: Engineering strategy of yeast metabolism for higher alcohol production. Microb Cell Fact 2011, 10(1):70

29. Boles $E$, de Jong-Gubbels P, Pronk JT: Identification and characterization of MAE1, the Saccharomyces cerevisiae structural gene encoding mitochondrial malic enzyme. J Bacteriol 1998, 180(11):2875-2882.

30. Ida Y, Furusawa C, Hirasawa T, Shimizu H: Stable disruption of ethanol production by deletion of the genes encoding alcohol dehydrogenase isozymes in Saccharomyces cerevisiae. J Biosci Bioeng 2012, 113(2):192-195.

31. Herrgard MJ, Swainston N, Dobson P, Dunn WB, Arga KY, Arvas M, Bluthgen $\mathrm{N}$, Borger $\mathrm{S}$, Costenoble $\mathrm{R}$, Heinemann $\mathrm{M}$, et al: A consensus yeast metabolic network reconstruction obtained from a community approach to systems biology. Nat Biotechnol 2008, 26(10):1155-1160.

32. Hohmann S: Characterization of $P D C 6$, a third structural gene for pyruvate decarboxylase in Saccharomyces cerevisiae. J Bacteriol 1991, 173(24):7963-7969.

33. Oud B, Flores CL, Gancedo C, Zhang X, Trueheart J, Daran JM, Pronk JT, van Maris AJ: An internal deletion in MTH1 enables growth on glucose of pyruvate-decarboxylase negative, non-fermentative Saccharomyces cerevisiae. Microb Cell Fact 2012, 11:131.

34. Ishii J, Izawa K, Matsumura S, Wakamura K, Tanino T, Tanaka T, Ogino C, Fukuda $\mathrm{H}$, Kondo $\mathrm{A}$ : A simple and immediate method for simultaneously evaluating expression level and plasmid maintenance in yeast. $J$ Biochem 2009, 145(6):701-708.

35. Ito H, Fukuda $Y$, Murata K, Kimura A: Transformation of intact yeast cells treated with alkali cations. J Bacteriol 1983, 153(1):163-168.

36. Katahira S, Mizuike A, Fukuda H, Kondo A: Ethanol fermentation from lignocellulosic hydrolysate by a recombinant xylose- and cellooligosaccharide-assimilating yeast strain. Appl Microbiol Biotechnol 2006, 72(6):1136-1143.

doi:10.1186/1475-2859-12-119

Cite this article as: Matsuda et al.: Increased isobutanol production in Saccharomyces cerevisiae by eliminating competing pathways and resolving cofactor imbalance. Microbial Cell Factories 2013 12:119.

\section{Submit your next manuscript to BioMed Central and take full advantage of:}

- Convenient online submission

- Thorough peer review

- No space constraints or color figure charges

- Immediate publication on acceptance

- Inclusion in PubMed, CAS, Scopus and Google Scholar

- Research which is freely available for redistribution

Submit your manuscript at www.biomedcentral.com/submit
C Biomed Central 